\title{
RESPOSTA AO CALCÁRIO E AO FÓSFORO DE GENÓTIPOS DE TRIGO COM DIFERENTES TOLERÂNCIAS AO ALUMÍNIO'
}

\author{
JOSÉ GUILHERME DE FREITAS², HEITOR CANTARELLA ${ }^{3}$, CARLOS EDUARDO DE OLIVEIRA CAMARGO², \\ ANTONIO WILSON PENTEADO FERREIRA FILHO ${ }^{4}$, ARMANDO PETTINELLI JÚNIOR ${ }^{5}$, \\ VALDIR JOSUÉ RAMOS ${ }^{6}$ e JOÃO CARLOS FELICIO ${ }^{4}$
}

\begin{abstract}
RESUMO - O objetivo do trabalho foi avaliar a resposta, à calagem e ao fósforo, de quatro genótipos de trigo com diferentes tolerâncias à acidez do solo. Os experimentos foram instalados de 1987 a $1995 \mathrm{em}$ um cambissolo ácido e de baixa fertilidade, sem irrigação e após pousio ou soja. O delineamento foi de blocos, em parcela subsubdividida, com quatro repetições. A parcela constou de três doses de calcário $\left(0,6,5\right.$ e $\left.13 \mathrm{t} \mathrm{ha}^{-1}\right)$; a subparcela, de três doses de $\mathrm{P}\left(0,30\right.$ e $90 \mathrm{~kg} \mathrm{ha}^{-1}$ de $\left.\mathrm{P}_{2} \mathrm{O}_{5}\right)$, e as subsubparcelas, de quatro genótipos (IAC-5, IAC-24, IAC-60 e Anahuac). O calcário foi aplicado no primeiro ano (1987) e reaplicado em 1991, e o $\mathrm{P}$, anualmente, no sulco de semeadura. Os genótipos de trigo tolerantes à acidez (IAC-5 e IAC-60) se mostraram mais responsivos à calagem e à aplicação de P e, mesmo em solos parcialmente corrigidos, apresentaram maiores rendimentos de grãos do que o material sensível à acidez (Anahuac). A correção da acidez e a adubação fosfatada provocaram a diminuição da relação palha:grão por favorecer mais a produção de grãos do que o desenvolvimento vegetativo. Essa relação foi menor e mais constante no genótipo IAC-60, tolerante à acidez. Os genótipos IAC-5 e IAC-60 mostraram-se mais eficientes em relação ao aproveitamento do P aplicado para o rendimento de grãos em solos de baixa fertilidade.
\end{abstract}

Termos para indexação: genótipo, calagem, adubação fosfatada.

\section{RESPONSE TO LIME AND PHOSPHORUS OF WHEAT GENOTYPES WITH DIFFERENT TOLERANCE TO ALUMINIUM}

\begin{abstract}
Field experiments were set up during nine years, in a low fertility, very acid Cambisol, with the objective of evaluating the response to lime and phosphorus of four wheat genotypes differing in tolerance to soil acidity. The experiments were carried out without irrigation, in randomized blocks with a split-split plot design, with four replications. The plots contained the three rates of limestone $(0$, 6.5, and 13 ton ha- $\left.{ }^{-1}\right)$; the split-plots the rates of phosphorus $\left(0,30\right.$, and $90 \mathrm{~kg} \mathrm{ha}^{-1}$ of $\left.\mathrm{P}_{2} \mathrm{O}_{5}\right)$, and the split-split plots the wheat varieties (IAC-5, IAC-24, IAC-60 and Anahuac). Lime was applied in the first year (1987) and reapplied in 1991; phosphorus was applied yearly. Wheat varieties tolerant to soil acidity (IAC-5 and IAC-60) were more responsive to limestone and phosphorus application and, even in soils with acidity partially neutralized, showed higher grain yields than the acid sensitive variety (Anahuac). Limestone and phosphorus caused a decrease in the straw:grain ratio because they increased the grain yield more than the vegetative growth. This ratio was lower and more stable with IAC-60. Genotypes IAC-5 and IAC- 60 were the most efficient to use the applied phosphorus to produce grain. Yields of grain and straw, and plant size were usually more affected by phosphorus than by limestone application.
\end{abstract}

Index terms: genotype, liming, phosphorus fertilization.

\footnotetext{
${ }^{1}$ Aceito para publicação em 8 de abril de 1999.

${ }^{2}$ Eng. Agrôn., Dr., Centro de Plantas Graníferas, IAC, Caixa Postal 28, CEP 13001-970 Campinas, SP. E-mail: jfreitas@cec.iac.br

${ }^{3}$ Eng. Agrôn., Dr., Centro de Solos e Recursos Agroambientais, IAC.
}

\footnotetext{
${ }^{4}$ Eng. Agrôn., Centro de Plantas Graníferas, IAC.

${ }^{5}$ Eng. Agrôn., Estação Exp. Agr. Tatuí, Rod. Mario B. Mori SP 141, km 38, Caixa Postal 33, CEP 18270-000 Tatuí, SP.

${ }^{6}$ Eng. Agrôn., Dr., Estação Exp. Agr. Itararé, Est. Mun. ItararéBonsucesso, km 29,5, Caixa Postal 58, CEP 18460-000 Itararé, SP.
} 


\section{INTRODUÇÃO}

Muitos solos cultivados com trigo no Estado de São Paulo são ácidos e de baixa fertilidade, e limitam o rendimento de grãos. Assim, o uso de genótipos de trigo tolerantes ao Al tóxico e eficientes e responsivos ao aproveitamento de nutrientes (Bartlett \& Riego, 1972; Oliveira et al., 1984; Camargo et al., 1987; Miranda \& Rowell, 1987; Alves et al., 1988; Fageria, 1990; Huang \& Grunes, 1992; Ryan \& Kochian, 1993) assume importância na redução do custo de produção e no aumento do rendimento e qualidade dos grãos desse cereal.

O trigo cultivado no Brasil, quando estabelecido em sucessão a outras gramíneas, tem na deficiência do $\mathrm{N}$ o fator que mais limita o rendimento de grãos, seguida pela deficiência de P. Contudo, se a sucessão for a leguminosas, em particular à soja, a deficiência de $\mathrm{P}$ e a acidez do solo podem ser os fatores mais limitantes. Ao contrário do $\mathrm{N}$, o calcário e o $\mathrm{P}$ são recomendados, independentemente da cultura do trigo ser em condição de sequeiro ou irrigada, e da espécie cultivada anteriormente (Raij et al., 1996). Isto pode indicar que as respostas dos genótipos de trigo a estes nutrientes são mais estáveis do que ao N. As aplicações do calcário e P são baseadas na análise química do solo, enquanto o $\mathrm{N}$, não (Raij et al., 1996).

A cultura do trigo tem respondido de maneiras diferentes às aplicações de calcário e adubo fosfatado, em razão da cultivar, da água disponível e do tipo de solo, entre outros fatores (Leal \& Velloso, 1973; Magalhães, 1979; Camargo, 1984, 1985; Ben, 1989; Rosa \& Camargo, 1991; Batten, 1992; Ben \& Dechen, 1996; Souza, 1996). Oliveira et al. (1984) verificaram que entre os genótipos de trigo, o IAC-5 (origem nacional) foi mais eficiente na absorção do $\mathrm{P}$ e mais tolerante ao $\mathrm{Al}$ tóxico do que a variedade mexicana Alondra-46. Abichequer \& Bohnen (1998) também verificaram a maior eficiência na utilização de $\mathrm{P}$ pelo IAC-5, em relação aos genótipos de trigo IAS 20 e o CNT 8, em solução nutritiva. Souza (1996) verificou que o uso da calagem e/ou do $\mathrm{P}$ foi benéfico para as cultivares de trigo utilizadas, com relação aos rendimentos de massa da parte aérea e dos grãos. Entretanto, o efeito do $\mathrm{P}$ foi maior do que o da calagem para os rendimentos de grãos e de matéria seca e altura de plantas. Souza (1996) verificou que o genótipo de trigo BR-40, tolerante ao Al, poderia ser considerado mais eficiente e responsivo na absorção e utilização de nutrientes do solo, em comparação com o BR-36, sensível a esse elemento. O genótipo IAC-24 é também tolerante à acidez do solo (Felício et al., 1986; Camargo et al., 1992b).

Camargo (1984), comparando o efeito de níveis de $\mathrm{P}$ em solução nutritiva e em solo nos vasos sobre vários materiais de trigo, observou que o genótipo IAC-5 esteve sempre entre os mais eficientes na utilização desse elemento, o que foi observado também por Abichequer \& Bohnen (1998). Os demais genótipos testados têm pouca ou nenhuma importância comercial no presente. O mesmo comportamento do IAC-5 foi observado por Ben \& Rosa (1983), em comparação com materiais cultivados no sul do Brasil.

Poucas informações estão disponíveis em relação ao comportamento de genótipos mais cultivados atualmente para o Estado de São Paulo em relação à resposta a $\mathrm{P}$ em diferentes condições de acidez do solo. Os objetivos deste trabalho foram: estudar as respostas, ao calcário e ao $\mathrm{P}$, de quatro cultivares de trigo distintas quanto à tolerância à acidez do solo, com vistas à produtividade de grãos e palha e altura das plantas; identificar, entre os genótipos avaliados, os mais eficientes na absorção e utilização do $\mathrm{P}$ e do calcário aplicados, e avaliar as interações significativas existentes entre as doses de calcário e de $\mathrm{P}$ e os genótipos de trigo.

\section{MATERIAL E MÉTODOS}

Os experimentos foram instalados anualmente no mesmo local, num total de nove ensaios, na Estação Experimental de Itararé, Instituto Agronômico (IAC), SP, latitude $24^{0} 15^{\prime} \mathrm{S}$, longitude $49^{0} 15^{\prime} \mathrm{W}$ e altitude $1.150 \mathrm{~m}$, no período de 1987 a 1995. A Estação Experimental de Itararé está localizada na zona tritícola B, a qual se caracteriza pelo clima semitemperado (C.F.B.) Köppen, com $674 \mathrm{~mm}$ de precipitação pluvial de março a setembro, com temperaturas anuais média, máxima e mínima de 17,0, 21,0 e $12,3^{\circ} \mathrm{C}$, respectivamente. As temperaturas são mais baixas e o balanço hídrico é mais favorável no período de outono-inverno, época do cultivo de trigo, em Itararé do que no resto do Estado de São Paulo; e portanto não houve estresse hídrico. O solo do experimento é um 
Cambissolo álico, fase urutu, muito ácido e de baixa fertilidade (Comissão Técnica de Trigo, 1996). O experimento foi conduzido sob condição de sequeiro e em sucessão ao pousio (1987 a 1988) e à soja (1989 a 1995). O delineamento estatístico utilizado foi o de blocos ao acaso, no esquema fatorial de parcela subsubdividida, com quatro repetições. A parcela foi constituída de três doses de calcário dolomítico $\left(0,6,5\right.$ e 13 t ha ${ }^{-1}$, aplicadas em 1987 e reaplicadas nas mesmas quantidades em 1991); a dose de $13 \mathrm{t} \mathrm{ha}^{-1}$ foi escolhida por ser a necessária para atingir, por cálculo, $70 \%$ da saturação por base. A subparcela foi constituída por três doses de P $(0,30$ e $90 \mathrm{~kg}$ de $\mathrm{P}_{2} \mathrm{O}_{5}$ ha $^{-1}$, aplicadas todos os anos), como superfosfato simples, e as subsubparcelas, por quatro genótipos de trigo (IAC-5, IAC-24 e IAC-60 de origem nacional e tolerantes ao $\mathrm{Al}$ e Anahuac, de origem mexicana e sensível ao Al, conforme Camargo et al. (1995). Esses genótipos de trigo foram os selecionados, por serem os mais cultivados no Estado de São Paulo, na época.

A densidade de semeadura foi de 80 sementes viáveis por metro linear. As análises do solo foram feitas com base na metodologia de Raij \& Quaggio (1983), e as de plantas, na de Bataglia et al. (1978). A dose de $90 \mathrm{~kg} \mathrm{ha}^{-1} \mathrm{de}$ $\mathrm{P}_{2} \mathrm{O}_{5}$ corresponde à máxima recomendada no Estado de São Paulo (Raij et al., 1996). As doses anuais de $\mathrm{N} \mathrm{e} \mathrm{K}_{2} \mathrm{O}$ foram 60 e $30 \mathrm{~kg} \mathrm{ha}^{-1}$, respectivamente, nas formas de uréia e cloreto de potássio. Os micronutrientes $\mathrm{B}$ e $\mathrm{Zn}$ foram aplicados anualmente nas doses de 550 e $1.100 \mathrm{~g} \mathrm{ha}^{-1}$, respectivamente, nas formas de bórax e sulfato de zinco. A adubação nitrogenada, na forma de uréia, foi parcelada, com aplicações de $20 \mathrm{~kg} \mathrm{ha}^{-1}$ de $\mathrm{N}$, por ocasião da semeadura, juntamente com os outros nutrientes, e $40 \mathrm{~kg} \mathrm{ha}^{-1}$ em cobertura, no estádio de alongamento (Z-30) (Large, 1954), ou seja, 40 dias após a emergência das plântulas de trigo. $\mathrm{O}$ controle dos fungos foi feito com pulverizações de propiconazole conforme a Comissão Técnica de Trigo (1996).

As subsubparcelas foram constituídas por sete linhas de $3 \mathrm{~m}$ de comprimento, a espaços de $0,2 \mathrm{~m}$, sendo as cinco linhas centrais colhidas para quantificar os rendimentos de grãos e de palha da parte aérea, colhida $3 \mathrm{~cm}$ acima do nível do solo. A altura das plantas foi medida da superfície do solo até a extremidade da espiga, com exceção das aristas. Foram feitas sete colheitas no período, pois nos anos 1990 e 1994 não houve produção de grãos, devido a geadas.

As análises individuais e ou conjuntas da variância foram feitas com o programa SANEST - Sistema de Análise Estatística (Zonta et al., 1987). As análises conjuntas foram obtidas após a verificação das homogeneidades das variâncias dos erros das subsubparcelas. A homogeneidade das variâncias dos resíduos das subsubparcelas foi calculada dividindo-se os maiores erros das análises individuais pelos menores resíduos e multiplicando por 2; se o valor encontrado for menor ou igual a sete, é possível realizar as análises conjuntas, conforme Cochran \& Cox (1957). Utilizou-se o teste de Tukey, a $5 \%$ de probabilidade, para comparar as médias entre genótipos, utilizando-se as variâncias complexas calculadas, dentro de cada dose de calcário e de $\mathrm{P}$, conforme Cochran \& Cox (1957). As superfícies de resposta foram ajustadas com os dados dos sete anos por meio do sistema de ajuste de equações da planilha eletrônica Excel 7.0 (Microsoft Corp. Redmond, WA, EUA). Os cálculos das doses mais econômicas de calcário e de $\mathrm{P}$, bem como as taxas de substituição desses insumos para a produção de uma mesma quantidade de grãos de trigo (isoquantas das superfícies de respostas) seguiram os procedimentos descritos por Black (1992). Para isso, foram empregadas as equações das superfícies de resposta e considerados os seguintes preços: calcário $=\mathrm{R} \$ 0,040 / \mathrm{kg} ; \mathrm{N}=\mathrm{R} \$ 0,80 / \mathrm{kg}$; $\mathrm{P}_{2} \mathrm{O}_{5}=\mathrm{R} \$ 0,80 / \mathrm{kg}$ e trigo $=\mathrm{R} \$ 0,18 / \mathrm{kg}$.

\section{RESULTADOS E DISCUSSÃO}

$\mathrm{Na}$ análise conjunta, foram verificados efeitos significativos a $5 \%$ de probabilidade pelo teste $\mathrm{F}$ em relação a doses de calcário, de $\mathrm{P}$, genótipos e interações entre esses fatores em relação às produtividades de grãos e de palha e à altura das plantas. Assim, foi possível fazer o desdobramento da interação tripla, para observar as possíveis diferenças significativas.

Os efeitos de doses de calcário ou de $\mathrm{P}$ foram avaliados por meio de curvas ou superfícies de resposta. $\mathrm{O}$ fator $\mathrm{P}$ foi o que mais afetou os rendimentos de grão e de palha e a altura de plantas do trigo (Tabela 1), com efeito quadrático significativo. Esses resultados estão de acordo com os obtidos por Souza (1996).

$\mathrm{O}$ incremento de produção observado quando a dose de $\mathrm{P}$ passou de 30 para $90 \mathrm{~kg} \mathrm{ha}^{-1}$ de $\mathrm{P}_{2} \mathrm{O}_{5}$ diminuiu, à medida que os níveis de calcário aumentaram. Esse efeito de interação há muito tem sido observado com várias culturas (Vidor \& Freire, 1972; Silva et al., 1980), muitas vezes de modo mais intenso que o obtido no presente estudo.

Nas parcelas onde não se aplicou calcário ou $\mathrm{P}$ as plantas apresentaram um crescimento e desenvolvi- 
TABELA 1. Rendimento de palha e de grãos e altura dos genótipos de trigo, dentro de cada dose de calcário (C) e fósforo (P). Médias de sete anos ${ }^{1}$.

\begin{tabular}{|c|c|c|c|c|c|c|c|c|c|c|}
\hline \multirow[t]{4}{*}{ Genótipo } & \multicolumn{9}{|c|}{ Calcário $\left(\mathrm{t} \mathrm{ha}^{-1}\right)$} & \multirow[t]{4}{*}{ Média } \\
\hline & \multicolumn{3}{|c|}{0} & \multicolumn{3}{|c|}{6,5} & \multicolumn{3}{|c|}{13} & \\
\hline & \multicolumn{3}{|c|}{$\mathrm{P}_{2} \mathrm{O}_{5}\left(\mathrm{~kg} \mathrm{ha}^{-1}\right)$} & \multicolumn{3}{|c|}{$\mathrm{P}_{2} \mathrm{O}_{5}\left(\mathrm{~kg} \mathrm{ha}^{-1}\right)$} & \multicolumn{3}{|c|}{$\mathrm{P}_{2} \mathrm{O}_{5}\left(\mathrm{~kg} \mathrm{ha}^{-1}\right)$} & \\
\hline & 0 & 30 & 90 & 0 & 30 & 90 & 0 & 30 & 90 & \\
\hline \multicolumn{11}{|c|}{ Rendimento de palha $\left(\mathrm{t} \mathrm{ha}^{-1}\right)$} \\
\hline IAC-5 & $0,61 \mathrm{a}$ & $1,25 \mathrm{a}$ & $3,43 b$ & $1,83 \mathrm{~b}$ & $3,42 \mathrm{a}$ & $4,24 \mathrm{ab}$ & $1,95 \mathrm{a}$ & $3,76 \mathrm{a}$ & $4,63 \mathrm{a}$ & $2,47 b$ \\
\hline IAC-24 & $0,31 b$ & $1,4 \mathrm{a}$ & $2,91 \mathrm{c}$ & $2,04 \mathrm{ab}$ & $3,14 b$ & $4,00 \mathrm{~b}$ & $2,15 \mathrm{a}$ & $2,93 b$ & $3,60 \mathrm{c}$ & $1,53 b$ \\
\hline IAC-60 & $0,36 \mathrm{ab}$ & $1,27 \mathrm{a}$ & $3,94 \mathrm{a}$ & $2,15 \mathrm{a}$ & $3,53 \mathrm{a}$ & $4,31 \mathrm{a}$ & $1,99 \mathrm{a}$ & $3,83 \mathrm{a}$ & $4,19 b$ & $2,90 \mathrm{a}$ \\
\hline Anahuac & $0,04 \mathrm{c}$ & $0,39 b$ & $1,45 \mathrm{~d}$ & $1,13 \mathrm{c}$ & $1,91 \mathrm{c}$ & $2,56 \mathrm{c}$ & $1,09 b$ & $2,02 \mathrm{c}$ & $2,91 \mathrm{~d}$ & $1,69 \mathrm{c}$ \\
\hline Média P & 0,11 & 1,05 & $3,03 \mathrm{~L}$ & 1,86 & 2,58 & $4,04 \mathrm{~L}$ & 1,81 & 3,22 & $4,28 \mathrm{Q}$ & \\
\hline Média C & & & 1,38 & & & 2,83 & & & $3,06 \mathrm{Q}$ & \\
\hline \multicolumn{11}{|c|}{ Rendimento de grãos $\left(\mathrm{t} \mathrm{ha}^{-1}\right)$} \\
\hline IAC-5 & $0,08 \mathrm{ab}$ & $0,37 \mathrm{ab}$ & $0,99 b$ & $0,45 b$ & $1,18 \mathrm{a}$ & $1,46 b$ & $0,53 b$ & $1,21 b$ & $1,54 \mathrm{~b}$ & $0,87 b$ \\
\hline IAC-24 & $0,05 \mathrm{ab}$ & $0,30 \mathrm{~b}$ & $0,64 \mathrm{c}$ & $0,32 \mathrm{c}$ & $0,88 b$ & $1,27 \mathrm{c}$ & $0,37 \mathrm{c}$ & $1,02 \mathrm{c}$ & $1,18 \mathrm{c}$ & $0,67 \mathrm{c}$ \\
\hline IAC-60 & $0,11 \mathrm{a}$ & $0,45 \mathrm{a}$ & $1,18 \mathrm{a}$ & $0,58 \mathrm{a}$ & $1,24 \mathrm{a}$ & $1,71 \mathrm{a}$ & $0,66 \mathrm{a}$ & $1,50 \mathrm{a}$ & $1,66 \mathrm{a}$ & $1,01 \mathrm{a}$ \\
\hline Anahuac & $0 \mathrm{c}$ & $0,10 \mathrm{c}$ & $0,43 \mathrm{~d}$ & $0,21 d$ & $0,59 \mathrm{c}$ & $0,84 d$ & $0,28 \mathrm{~d}$ & $0,70 \mathrm{~d}$ & $1,02 d$ & $0,46 \mathrm{~d}$ \\
\hline Média P & 0,06 & 0,30 & $0,81 \mathrm{~L}$ & 0,39 & 0,97 & $1,32 \mathrm{Q}$ & 0,46 & 1,11 & $1,35 \mathrm{Q}$ & \\
\hline Média C & & & 0,39 & & & 0,89 & & & $0,97 \mathrm{Q}$ & \\
\hline \multicolumn{11}{|c|}{ Altura da planta $(\mathrm{cm})$} \\
\hline IAC-5 & $23,9 \mathrm{a}$ & $71,7 \mathrm{a}$ & $98,4 \mathrm{a}$ & $71,5 \mathrm{a}$ & $103,5 \mathrm{a}$ & $111,9 \mathrm{a}$ & $75,9 a$ & $100,8 \mathrm{a}$ & $113,2 \mathrm{a}$ & $85,7 \mathrm{a}$ \\
\hline IAC-24 & $13,8 \mathrm{bc}$ & $52,8 \mathrm{~b}$ & $67,8 \mathrm{c}$ & $53,3 \mathrm{c}$ & $70,3 c$ & $78,9 \mathrm{c}$ & $54,3 \mathrm{c}$ & $71,2 \mathrm{c}$ & $75,3 \mathrm{c}$ & $59,8 \mathrm{c}$ \\
\hline IAC-60 & $18,7 \mathrm{ab}$ & $58,1 \mathrm{~b}$ & $76,8 \mathrm{~b}$ & $62,6 b$ & $80,8 \mathrm{~b}$ & $85,5 b$ & $61,2 b$ & $81,9 b$ & $87,2 b$ & $68,1 \mathrm{~b}$ \\
\hline Anahuac & $9,7 \mathrm{c}$ & $42,3 \mathrm{c}$ & $55,5 \mathrm{~d}$ & $51,3 \mathrm{c}$ & $67,3 \mathrm{c}$ & $68,9 \mathrm{~d}$ & $53,0 \mathrm{c}$ & $66,3 c$ & $67,4 d$ & $53,5 \mathrm{~d}$ \\
\hline Média P & 16,5 & 56,2 & $74,6 \mathrm{Q}$ & 59,7 & 80,5 & $86,3 \mathrm{Q}$ & 61,1 & 80,1 & $85,8 \mathrm{Q}$ & \\
\hline Média C & & & 49,1 & & & 75,5 & & & $75,6 \mathrm{Q}$ & \\
\hline
\end{tabular}

${ }^{1}$ Médias seguidas pela mesma letra na coluna, não diferem entre si, a 5\% de probabilidade, pelo teste de Tukey; L e Q: efeitos linear e quadrático significativo a $5 \%$ de probabilidade pelo teste $\mathrm{t}$.

mento muito reduzido, e as produções foram inexpressivas, o que evidencia as sérias limitações de fertilidade do solo utilizado (Tabela 1). As aplicações anuais de $\mathrm{P}$ e as duas calagens realizadas em 1987 e 1991 pouco afetaram o conteúdo do P (extraído pelo método da resina) na camada arável do solo (Tabela 2) ao contrário do que tem sido observado em vários experimentos com outras culturas (Raij \& Quaggio, 1990). A textura leve (abaixo de 35\% de argila no horizonte A) e precipitações relativamente altas (1.250 mm anuais) devem ter contribuído para uma intensa lixiviação de bases, ocorrência já observada em outra ocasião, neste mesmo solo, por Quaggio et al. (1991). Um ano após a calagem, as saturações por bases das parcelas que receberam 6,5 e 13 t ha $^{-1}$ de calcário decresceram de $50 \%$ para $13 \%$ e de $63 \%$ para $36 \%$, respectivamente. Apesar do pequeno efeito residual dos tratamentos aplicados, foi possível manter um gradiente de acidez, o qual, combinado com as doses anuais de P, permitiu avaliar o comportamento dos genótipos em razão das variáveis estudadas.

As respostas quadráticas (ao invés de efeitos predominantemente lineares) à calagem e ao $\mathrm{P}$ nesse Cambissolo de limitada fertilidade, podem ter ocorrido em virtude de provável limitação de outros nutrientes, especialmente de $\mathrm{N}$ e $\mathrm{K}$. As quantidades desses elementos aplicadas foram calculadas com 
base nas respostas obtidas em outros locais do Estado de São Paulo (Camargo et al., 1992a) com menores problemas de fertilidade.

Os vários genótipos responderam de modo diferente à aplicação de calcário e de P. De modo geral, o genótipo IAC-60 apresentou os maiores rendimentos de grãos em condições de ausência ou baixas doses dos tratamentos, porém com pequena diferença, nem sempre significativa em relação ao IAC-5. A cultivar Anahuac apresentou crescimento e rendimento de grãos e palha inferiores às demais; a IAC-24 teve comportamento intermediário (Tabela 1). A maior eficiência na utilização do $\mathrm{P}$ pelo genótipo de trigo IAC-5, também foi verificada por Abichequer \& Bohnen (1998).

Apesar de mais tolerantes à acidez, os genótipos IAC-60 e IAC-5 se mostraram também responsivos à calagem, e, mesmo no tratamento que recebeu $13 \mathrm{t} \mathrm{ha}^{-1}$ do corretivo, foram mais produtivos que o material mais sensível (Tabela 1). Comportamento semelhante ocorreu em relação ao $\mathrm{P}$.

A aplicação de calcário e de P provocou grande redução da relação palha:grão. Essa variou de 5,7 na ausência de ambos os tratamentos, para 3,6 apenas, com a adição de $\mathrm{P}, 3,9$ com a aplicação de $13 \mathrm{tha}^{-1} \mathrm{de}$ calcário e 2,8 quando ambos os insumos foram aplicados nas doses máximas. Isso mostra que as condições de acidez e de deficiência de $\mathrm{P}$ afetam mais o rendimento de grãos do que a de matéria seca da parte aérea, ou seja, afetam mais o crescimento e desenvolvimento reprodutivo do que o vegetativo, o que está de acordo com Souza (1996).

A relação palha:grão foi menor e mais constante com o IAC-60 (variou de 3,7 a 2,5) do que com os demais materiais. Em geral, os valores obtidos com os materiais mais tolerantes à acidez foram menores do que no material sensível. Além disso, a relação palha:grão foi mais variável com o IAC-24 do que com o Anahuac, principalmente porque a calagem $\mathrm{e}$ o $\mathrm{P}$ provocaram aumentos proporcionalmente maiores na produção de palha no primeiro genótipo do que no segundo (Tabela 1).

Os rendimentos de grãos variaram de ano para ano, de acordo com as condições climáticas, e, aparentemente, de acordo com o período de aplicação de calcário (Fig. 1). Para maior concisão, na Fig. 1 são apresentados apenas os dados de anos selecionados e dos genótipos com comportamentos mais contrastantes (IAC-60 e Anahuac). Os dados obtidos com IAC-5 e IAC-24 foram semelhantes aos do IAC-60. As respostas ao calcário e ao $\mathrm{P}$ foram relativamente pequenas no primeiro ano (1987), mas aumentaram em 1988 e 1990 . Após a reaplicação do calcário em 1991, ocorreu a mesma tendência (Fig. 1). As respostas observadas com o genótipo IAC-60 foram, de modo geral, semelhantes às conseguidas com o Anahuac, embora a magnitude de respostas tenha sido maior em IAC-60. No período avaliado, a resposta ao $\mathrm{P}$ foi maior do que à calagem; tendência mais acentuada em Anahuac do que em IAC-60 (Fig. 1).

TABELA 2. Resultados de análises do solo, na profundidade de 0-20 $\mathrm{cm}$ de fósforo, cálcio, magnésio e saturação por bases (V) dentro de cada dose de calcário em anos selecionados. Médias de quatro repetições.

\begin{tabular}{|c|c|c|c|c|c|c|c|c|c|c|c|c|}
\hline \multirow{3}{*}{$\begin{array}{c}\text { Ano de } \\
\text { amostragem }\end{array}$} & \multicolumn{12}{|c|}{ Calcário $\left(\mathrm{t} \mathrm{ha}^{-1}\right)$} \\
\hline & \multicolumn{4}{|c|}{0} & \multicolumn{4}{|c|}{6,5} & \multicolumn{4}{|c|}{13} \\
\hline & $\mathrm{P}$ & $\mathrm{Ca}$ & $\mathrm{Mg}$ & $\mathrm{V}$ & $\mathrm{P}$ & $\mathrm{Ca}$ & $\mathrm{Mg}$ & $\mathrm{V}$ & $\mathrm{P}$ & $\mathrm{Ca}$ & $\mathrm{Mg}$ & $\mathrm{V}$ \\
\hline & $\left(\mathrm{mg} \mathrm{dm}^{-3}\right)$ & $(\mathrm{mm}$ & $\left(m^{-3}\right)$ & $(\%)$ & $\left(\mathrm{mg} \mathrm{dm}^{-3}\right)$ & $(\mathrm{mm}$ & $\left.\mathrm{dm}^{-3}\right)$ & $(\%)$ & $\left(\mathrm{mg} \mathrm{dm}^{-3}\right)$ & $(\mathrm{mm}$ & $\left.\mathrm{dm}^{-3}\right)$ & $(\%)$ \\
\hline $1987^{1}$ & 4 & 2 & 2 & 4 & - & - & - & - & - & - & - & - \\
\hline $1987^{2}$ & 4 & 2 & 2 & 5 & 3 & 27 & 32 & 50 & 4 & 53 & 57 & 63 \\
\hline 1988 & 2 & 2 & 2 & 3 & 3 & 7 & 7 & 13 & 3 & 21 & 20 & 36 \\
\hline 1990 & 5 & 4 & 2 & 4 & 4 & 14 & 9 & 15 & 6 & 23 & 19 & 37 \\
\hline 1992 & 5 & 4 & 2 & 4 & 7 & 12 & 10 & 14 & 6 & 37 & 27 & 39 \\
\hline 1994 & 6 & 3 & 2 & 4 & 6 & 13 & 11 & 19 & 5 & 20 & 15 & 33 \\
\hline
\end{tabular}

${ }^{1}$ Amostragem antes da calagem, do preparo do solo e da semeadura do trigo.

${ }^{2}$ Amostragem 6 meses depois da calagem, do preparo do solo e da semeadura do trigo. 

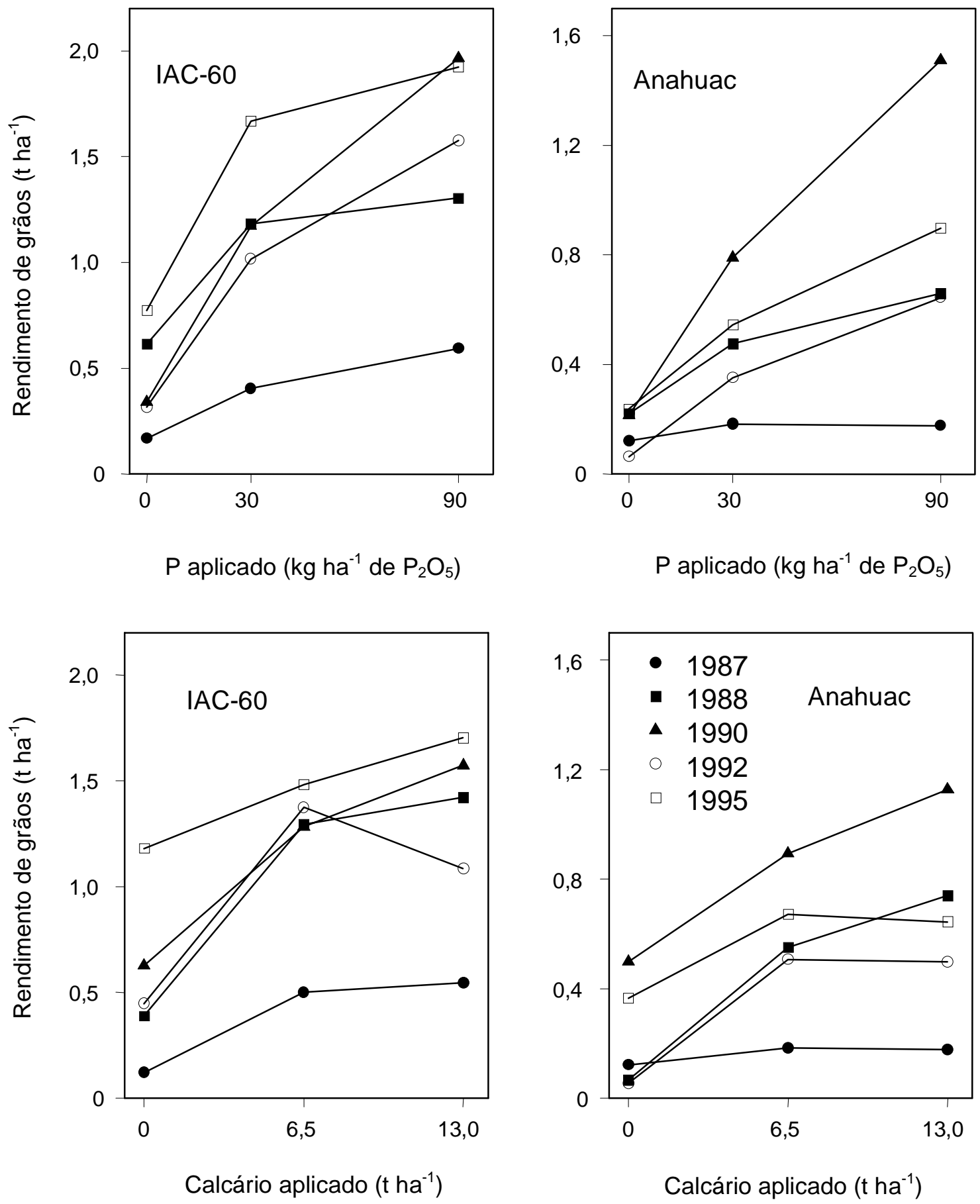

FIG. 1. Resposta dos genótipos de trigo IAC-60 e Anahuac ao calcário e ao fósforo, em relação ao rendimento de grãos. Médias de cinco anos. 
Equações de superfícies de resposta de rendimento de grãos de trigo em razão das doses de calcário e $\mathrm{P}$ aplicadas, calculadas com base na média de sete anos de produção, são apresentadas na Tabela 3 . Os ajustes obtidos foram razoáveis, considerandose as naturezas dos dados; os coeficientes de determinação variaram de 0,51 a 0,60 . Os coeficientes lineares relativos a doses de calcário e de $\mathrm{P}$ indicam que os materiais tolerantes à acidez obtiveram maiores rendimentos de grãos por unidade de insumo do que o material sensível, e, especialmente, do que o genótipo Anahuac. O efeito das interações entre calcário e $\mathrm{P}$ foi pequeno (e negativo) para os materiais obtidos no Brasil, mas foi relativamente importante (e positivo) para o Anahuac (Tabela 3). A provável razão para essa diferença é a maior tolerância à acidez nos materiais de trigo desenvolvidos no IAC.

Com base nas superfícies de respostas da Tabela 3, foi possível estimar a quantidade de $\mathrm{P}$ necessária para produzir $1 \mathrm{t} \mathrm{ha}^{-1}$ de trigo no Cambissolo da área do experimento, simulando o tratamento com várias doses de calcário (Tabela 4). A opção mais econômica para os materiais tolerantes à acidez seria utilizar 3,25 $\mathrm{t} \mathrm{ha}^{-1}$ de calcário e as doses de $\mathrm{P}$ indicadas na Tabela 4. O custo dessas opções é aproximadamente $1 / 3$ do custo para produzir $1 \mathrm{t} \mathrm{ha}^{-1}$ de trigo Anahuac (13 t ha ${ }^{-1}$ de calcário e $79 \mathrm{~kg} \mathrm{ha}^{-1}$ de $\mathrm{P}_{2} \mathrm{O}_{5}$ ). Os dados da Tabela 4 mostram que a eficiência do uso do P aplicado e o aproveitamento do efeito da calagem para a produção de grãos de trigo são bastante aumentados com o uso de genótipos adequados às condições do ambiente e às limitações do solo. Assim, para se obter um mesmo rendimento de grãos $\left(1 \mathrm{t} \mathrm{ha}^{-1}\right)$ no solo tratado com $6,5 \mathrm{t} \mathrm{ha}^{-1} \mathrm{de}$ calcário, foram necessários 18 e $26 \mathrm{~kg} \mathrm{ha}^{-1}$ de $\mathrm{P}_{2} \mathrm{O}_{5}$ para o IAC-60 e IAC-5 respectivamente, e $47 \mathrm{~kg} \mathrm{ha}^{-1}$ de $\mathrm{P}_{2} \mathrm{O}_{5}$ para o IAC-24. Com esse nível de calagem não se conseguiu o rendimento de grãos especificado com o genótipo Anahuac, mesmo com a aplicação de $90 \mathrm{~kg} \mathrm{ha}^{-1}$ de $\mathrm{P}_{2} \mathrm{O}_{5}$ (Tabela 4).

A altura das plantas do trigo teve um comportamento semelhante ao do rendimento de grãos, ou seja, as respostas ao calcário e ao $\mathrm{P}$ foram quadráticas. Independentemente das doses de calcário e de $\mathrm{P}$, os genótipos de trigo mantiveram a variabilidade genética quanto à altura das plantas (Tabela 1). Também houve alta correlação entre rendimento de grãos e altura das plantas de trigo.

Os resultados de análise da folha-bandeira dos genótipos IAC-60 e Anahuac (Tabela 5) indicam que

TABELA 3. Superfícies de respostas do rendimento de grãos de quatro genótipos de trigo em razão da aplicação de calcário e de fósforo. Médias de sete anos.

\begin{tabular}{llc}
\hline Genótipo & \multicolumn{1}{c}{ Equação $^{1}$} & $\mathrm{R}^{2}$ \\
\hline IAC-5 & $\mathrm{Y}=-25,46+114,03 \mathrm{C}-5,2070 \mathrm{C}^{2}+23,31 \mathrm{P}-0,1371 \mathrm{P}^{2}-0,0167 \mathrm{CP}$ & 0,58 \\
IAC-24 & $\mathrm{Y}=-63,80+107,58 \mathrm{C}-5,4753 \mathrm{C}^{2}+19,13 \mathrm{P}-0,1224 \mathrm{P}^{2}-0,0984 \mathrm{CP}$ & 0,57 \\
IAC-60 & $\mathrm{Y}=-10,80+135,99 \mathrm{C}-5,9753 \mathrm{C}^{2}+24,54 \mathrm{P}-0,1379 \mathrm{P}^{2}-0,0869 \mathrm{CP}$ & 0,60 \\
Anahuac & $\mathrm{Y}=-62,10+66,88 \mathrm{C}-2,9184 \mathrm{C}^{2}+10,17 \mathrm{P}-0,0557 \mathrm{P}^{2}-0,2307 \mathrm{CP}$ & 0,51 \\
\hline
\end{tabular}

${ }^{1}$ Y: rendimento de grãos $\left(\mathrm{kg} \mathrm{ha}^{-1}\right)$; C: calcário aplicado em 1987 e reaplicado em 1991 ( $\left.\mathrm{tha}^{-1}\right)$; P: P aplicado anualmente $\left(\mathrm{kg} \mathrm{ha}^{-1}\right.$ de $\left.\mathrm{P}_{2} \mathrm{O}_{5}\right)$.

TABELA 4. Doses anuais de fósforo $\left(\mathrm{P}_{2} \mathrm{O}_{5}\right)$ necessárias para produzir $1,0 \mathrm{t} \mathrm{ha} \mathrm{h}^{-1}$ de grãos de trigo em solo com diferentes doses de calcário. Os cálculos foram realizados com base nas superfícies de resposta da Tabela 3. Médias de sete anos.

\begin{tabular}{lccccc}
\hline Genótipo & \multicolumn{5}{c}{ Calcário $\left(\mathrm{t} \mathrm{ha}^{-1}\right)$} \\
\cline { 2 - 6 } & 0 & 3,25 & 6,50 & 9,75 & 13,00 \\
\hline & & $\mathrm{P}_{2} \mathrm{O}_{5}\left(\mathrm{~kg} \mathrm{ha}^{-1}\right)$ & & \\
IAC-5 & - & 40 & 26 & 20 & 51 \\
IAC-24 & - & - & 47 & 41 & 12 \\
IAC-60 & 65 & 32 & 18 & 11 & 79 \\
Anahuac & - & - & - & - & \\
\hline
\end{tabular}


TABELA 5. Teores de alguns elementos na folha bandeira de trigo dos genótipos IAC-60 e Anahuac em razão das aplicações de calcário e fósforo. Médias de três anos.

\begin{tabular}{|c|c|c|c|c|c|c|c|}
\hline \multirow[t]{2}{*}{ Tratamento $^{1}$} & \multicolumn{7}{|c|}{ Teor do elemento } \\
\hline & $\mathrm{N}$ & $\mathrm{P}$ & $\mathrm{K}$ & $\mathrm{Ca}$ & $\mathrm{Mg}$ & $\mathrm{Mn}$ & $\mathrm{Al}$ \\
\hline & \multicolumn{7}{|c|}{ 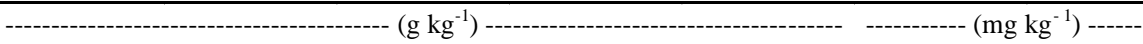 } \\
\hline & \multicolumn{7}{|c|}{ IAC-60 } \\
\hline $\mathrm{Calc}_{0} \mathrm{P}_{0}$ & 34,1 & 1,4 & 29,7 & 1,8 & 1,3 & 47,0 & 159,3 \\
\hline $\mathrm{Calc}_{0} \mathrm{P}_{30}$ & 32,6 & 1,8 & 29,2 & 2,3 & 1,1 & 55,8 & 44,3 \\
\hline $\mathrm{Calc}_{0} \mathrm{P}_{90}$ & 29,7 & 1,9 & 19,3 & 3,9 & 1,6 & 63,5 & 65,3 \\
\hline $\mathrm{Calc}_{6,5} \mathrm{P}_{0}$ & 32,6 & 1,5 & 26,8 & 2,3 & 2,0 & 27,5 & 59,3 \\
\hline Calc $_{6,5} \mathrm{P}_{30}$ & 31,5 & 1,8 & 19,7 & 3,0 & 2,3 & 41,8 & 50,5 \\
\hline Calc $_{6,5} \mathrm{P}_{90}$ & 32,0 & 2,1 & 20,5 & 3,8 & 2,3 & 40,5 & 39,5 \\
\hline $\mathrm{Calc}_{13} \mathrm{P}_{0}$ & 32,1 & 1,6 & 27,1 & 2,4 & 2,1 & 16,5 & 89,8 \\
\hline $\mathrm{Calc}_{13} \mathrm{P}_{30}$ & 29,5 & 1,8 & 21,8 & 3,1 & 2,4 & 22,0 & 41,5 \\
\hline \multirow[t]{2}{*}{ Calc $_{13} \mathrm{P}_{90}$} & 31,0 & 2,2 & 16,1 & 3,8 & 3,0 & 25,5 & 31,8 \\
\hline & \multicolumn{7}{|c|}{ Anahuac } \\
\hline $\mathrm{Calc}_{0} \mathrm{P}_{0}$ & 19,2 & 1,0 & 10,8 & 1,8 & 0,9 & $--^{2}$ & $-^{2}$ \\
\hline $\mathrm{Calc}_{0} \mathrm{P}_{30}$ & 26,1 & 1,6 & 17,9 & 2,5 & 1,4 & 79,5 & 352,3 \\
\hline $\mathrm{Calc}_{0} \mathrm{P}_{90}$ & 25,0 & 1,8 & 15,8 & 3,3 & 1,6 & 51,3 & 202,0 \\
\hline Calc $_{6,5} \mathrm{P}_{0}$ & 27,9 & 1,4 & 21,4 & 2,5 & 2,1 & 30,0 & 278,5 \\
\hline Calc $_{6,5} \mathrm{P}_{30}$ & 25,1 & 1,5 & 20,3 & 2,3 & 1,9 & 32,8 & 138,0 \\
\hline Calc $_{6,5} \mathrm{P}_{90}$ & 23,6 & 1,9 & 17,0 & 2,9 & 2,1 & 25,8 & 76,5 \\
\hline $\mathrm{Calc}_{13} \mathrm{P}_{0}$ & 27,2 & 1,5 & 21,5 & 2,7 & 2,2 & 11,0 & 272,3 \\
\hline Calc $_{13} \mathrm{P}_{30}$ & 25,8 & 1,7 & 20,6 & 2,3 & 2,0 & 13,8 & 160,8 \\
\hline Calc $_{13} \mathrm{P}_{90}$ & 23,8 & 1,8 & 16,1 & 2,6 & 2,5 & 17,3 & 114,0 \\
\hline
\end{tabular}

${ }_{1}^{1}$ Os tratamentos são interações entre as doses de calcário (Calc) e as de $\mathrm{P}_{2} \mathrm{O}_{5}(\mathrm{P})$ utilizadas no estudo.

${ }^{2}$ Amostra insuficiente para análise.

as plantas cultivadas em parcelas sem calcário e $\mathrm{P}$ ou com o nível mais baixo usado para esses insumos, apresentavam teores foliares de $\mathrm{Ca}, \mathrm{Mg}$ e $\mathrm{P}$ abaixo do adequado para o trigo, de acordo com os dados compilados por Raij et al. (1996). A aplicação de calcário geralmente elevou os níveis foliares de Ca e $\mathrm{Mg}$, os quais passaram a se situar na parte inferior das faixas adequadas (2,5 a 10,0 $\mathrm{g} \mathrm{kg}^{-1}$ de Ca e 1,5 a $4,0 \mathrm{~g} \mathrm{~kg}^{-1} \mathrm{de} \mathrm{Mg}$ ). A adubação com $\mathrm{P}$ aumentou linearmente o teor foliar desse elemento, mas geralmente não foi suficiente para elevá-lo aos níveis adequados (2,1 a 3,3 $\mathrm{g} \mathrm{kg}^{-1}$ de P), especialmente no caso do genótipo Anahuac. Tal material apresentou concentrações de Al bem maiores do que as encontradas no genótipo tolerante à acidez (Tabela 5); essa é a diferença mais marcante dos resultados da análise foliar dos dois genótipos. As aplicações de calcário e de $\mathrm{P}$ provocaram uma redução no teor de Al foliar.

\section{CONCLUSÕES}

1. Genótipos de trigo tolerantes ao Al se mostram responsivos à calagem e à aplicação de $\mathrm{P}$.

2. A correção da acidez e a adubação fosfatada provocam a diminuição na relação palha:grão de trigo.

3. Os materiais de trigo tolerantes ao Al mostramse mais eficientes em relação ao aproveitamento do $\mathrm{P}$ aplicado.

4. Os teores foliares de Al são maiores no genótipo sensível a esse elemento no solo do que nos materiais tolerantes, e diminuem com a aplicação de adubo fosfatado ou de calcário.

\section{AGRADECIMENTO}

Ao Conselho Nacional de Desenvolvimento Científico e Tecnológico, CNPq, pela concessão de auxílio e bolsas de pesquisa. 


\section{REFERÊNCIAS}

ABICHEQUER, A.D.; BOHNEN, H. Eficiência de absorção, translocação e utilização de $\mathrm{P}$ por variedades de trigo. Revista Brasileira de Ciência do Solo, Viçosa, MG, v.22, n.1, p.21-26, 1998.

ALVES, V.M.C.; NOVAIS, R.F.; NEVES, J.C.L.; BARROS, N.F. Efeito do Al sobre absorção e translocação de $\mathrm{P}$ e sobre a composição mineral de duas cultivares de trigo. Pesquisa Agropecuária Brasileira, Brasília, v.23, n.6, p.563-573, 1988 .

BARTLETT, R.J.; RIEGO, D.C. Toxicity of hidroxi aluminum in relation to $\mathrm{pH}$ and phosphorus. Soil Science, Baltimore, v.114, n.3, p.194-200, 1972.

BATAGLIA, O.C.; TEIXEIRA, J.P.F; FURLANI, A.M.C.; GALLO, J.R. Métodos de análise química de plantas. Campinas : Instituto Agronômico, 1978. 31p. (Circular, 87)

BATTEN, G.D. A review of phosphorus efficiency in wheat. Plant and Soil, The Hague, v.146, p.163168, 1992

BEN, J.R. Resposta diferencial de genótipos de trigo (Triticum aestivum L.) a P no solo. Piracicaba : ESALQ/USP, 1989. 109p. Tese de Doutorado.

BEN, J. R.; DECHEN, A.R. Comportamento de genótipos de trigo em relação a P no solo. Revista Brasileira de Ciência do Solo, Campinas, v.20, p.77-82, 1996.

BEN, J.R.; ROSA, O.S. Comportamento de algumas cultivares de trigo em relação a $\mathrm{P}$ no solo. Pesquisa Agropecuária Brasileira, Brasília, v.18, n.9, p.967972, 1983

BLACK, C.A . Soil fertility evaluation and control. Boca Raton : Lewis, 1992. 746p.

CAMARGO, C.E.O. A concentração de P na tolerância de cultivares de trigo à toxicidade de $\mathrm{Al}$ em soluções nutritivas. Bragantia, Campinas, v.44, n.1, p.4964, 1985.

CAMARGO, C.E.O . Efeito de diferentes níveis de P em solução nutritiva e no solo no comportamento de cultivares de trigo. Bragantia, Campinas, v.43, n.1, p.63-86, 1984.

CAMARGO, C.E.O.; FELÍCIO, J.C.; FERREIRA FILHO, A.W.P.; FREITAS, J.G.; PETTINELLI JUNIOR, A. Adubação N, P, K para a cultura do trigo em condição de irrigação por aspersão. Cam- pinas : Instituto Agronômico, 1992a. 12p. (Boletim técnico, 145).

CAMARGO, C.E.O.; FELICIO, J.C.; FREITAS, J.G.; FERREIRA, A.W.P. Trigo duro: tolerância à toxicidade do Al, manganês e ferro em soluções nutritivas. Bragantia, Campinas, v.54, n.2, p.371-383, 1995.

CAMARGO, C.E.O.; FELÍCIO, J.C.; ROCHA, L.S. Trigo: tolerância ao Al em solução nutritiva. Bragantia Campinas, v.46, n.2, p.187-190, 1987.

CAMARGO, C.E.O.; SANTOS, R.R.; PETINELLI JUNIOR, A. Trigo duro: tolerância à toxicidade do $\mathrm{Al}$ em soluções nutritivas e no solo. Bragantia, Campinas, v.51, n.1, p.69-76, 1992b.

COCHRAN, W.G.; COX, G.M. Experimental designs 2.ed. New York : John Wiley, 1957. 618p.

COMISSÃO TÉCNICA DE TRIGO. Recomendações da Comissão Técnica de Trigo para 1996. Campinas : Instituto Agronômico, 1996. 68p. (Boletim técnico, 167).

FAGERIA, N.K. Response of wheat to phosphorus fertilization on an oxisol. Pesquisa Agropecuária Brasileira, Brasília, v.25, n.4, p.531-537, 1990.

FELICIO, J.C.; CAMARGO, C.E.O.; FREITAS, J.G.; FERREIRA, A.W.P.; BARROS, B.C.; CAMARGO, M.B.P. Avaliação de genótipos de trigo para o Vale do Paranapanema no quadriênio 1981-84. Bragantia, Campinas, v.45, n.2, p.257-277, 1986.

HUANG, J.W.; GRUNES, D.L. Potassium/ magnesium ratio effects on tolerance and mineral composition of wheat forage. Agronomy Journal, Madison, v.84, n.4, p.643-650, 1992.

LARGE, E.C. Growth stages in cereals. Illustration of the feekes scale. Plant Pathology, London, v.3, p.128129, 1954.

LEAL, J.R.; VELLOSO, A.C.C. Adsorção de fosfato em latossolos de cerrado. Pesquisa Agropecuária Brasileira, Brasília, v.8, n.1, p.81-88, 1973.

MAGALHÃES, J.C.A.J. Efeito de níveis de calcário e de $\mathrm{P}$ em duas variedades de trigo. Revista Brasileira de Ciência do Solo, Campinas, v.3, p.24-29, 1979.

MIRANDA, L.N.; ROWELL, D.L. The effects of lime and phosphorus on the function of wheat roots in acid topsoils in subsoils. Plant and Soil, The Hague, v.104, p.253-262, 1987. 
OLIVEIRA, O.F.; CAMARGO, C.E.O.; RAMOS, V.J Efeito do P sobre os componentes de produção, altura das plantas e rendimentos de grãos, em trigo. Bragantia, Campinas, v.43, n.1, p.31-44, 1984.

QUAGGIO, J.A.; RAMOS, V.J.; FURLANI, P.R.; CARELLI, M.L.C. Liming and molybdenum effects on nitrogen uptake and grain yield of corn. In: WRIGHT, R.J.; BALIGAR, V.C.; MURRNAN, R.P. (Eds.). Plant-soil interaction at low pH. Dordrecht : Kluwer Academic, 1991. p.327-332.

RAIJ, B. van; CANTARELLA, H.; QUAGGIO, J.A.; FURLANI, A.M.C. Recomendações de adubação e calagem para o Estado de São Paulo. Campinas : Instituto Agronômico, 1996. 290p. (Boletim técnico, 81).

RAIJ, B. van; QUAGGIO, J.A. Métodos de análise de solo para fins de fertilidade. Campinas : Instituto Agronômico, 1983.31p. (Boletim técnico, 81)

RAIJ, B. van; QUAGGIO, J.A. Extractable phosphorus availability indices as affected by liming. Communication in Soil Science and Plant Analysis, New York, v.21, n.13/16, p.1267-1276, 1990.

ROSA, O.S.; CAMARGO, C.E.O. Wheat breeding for better efficiency in phosphorus use. In: INTERNATIONAL CONFERENCE ON WHEAT
FOR NONTRADITIONAL WARM AREAS, 3., 1990, Foz do Iguaçu. Proceedings. México : CIMMYT, 1991. p333-351.

RYAN, P.R.; KOCHIAN, L.V. Interaction between aluminium toxicity and calcium uptake at the root apex in near-isogenic lines of wheat (Triticum aestivum L.) differing in aluminium tolerance. Plant Physiology, Bethesda, v.102, n.3, p.975-982, 1993.

SILVA, N.M. da; FERRAZ, C.A.M.; RODRIGUES FILHO, F.S.O.; HIROCE, R. O uso de calcário e superfosfato simples em algodão em um solo ácido argiloso. Bragantia, Campinas, v.39, p.39-50, 1980.

SOUZA, P.G. A Resposta diferencial à calagem e ao $\mathbf{P}$ de três cultivares de trigo (Triticum aestivum $\mathbf{L}$.) com diferentes graus de tolerância ao Al. Jaboticabal : FCAV, Universidade Estadual Paulista, 1996. 137p. Tese de Doutorado.

VIDOR, C.; FREIRE, J.R.J. Relação de substituição entre calcário e $\mathrm{P}$ aplicados ao solo na cultura da soja. Agronomia Sulriograndense, Porto Alegre, v.8, p.187-193, 1972.

ZONTA, E.P.; MACHADO, A.A.; SILVEIRA JUNIOR, P. Sistema de Análise Estatística para Microcomputadores. Manual de utilização. 2.ed. Pelotas : UFPel, 1987. 177p. 